\title{
Fuzzy-multi-mode Resource-constrained Discrete Time-cost- resource Optimization in Project Scheduling Using ENSCBO
}

\author{
Ali Kaveh $^{1 *}$, Farivar Rajabi ${ }^{1}$ \\ ${ }^{1}$ School of Civil Engineering, Iran University of Science and Technology, 16846-13114 Tehran, Iran \\ * Corresponding author, e-mail: alikaveh@iust.ac.ir
}

Received: 24 August 2021, Accepted: 12 September 2021, Published online: 17 September 2021

\begin{abstract}
Construction companies are required to employ effective methods of project planning and scheduling in today's competitive environment. Time and cost are critical factors in project success, and they can vary based on the type and amount of resources used for activities, such as labor, tools, and materials. In addition, resource leveling strategies that are used to limit fluctuations in a project's resource consumption also affect project time and cost. The multi-mode resource-constrained discrete-time-cost-resource optimization (MRC-DTCRO) is an optimization tool that is developed for scheduling of a set of activities involving multiple execution modes with the aim of minimizing time, cost, and resource moment. Moreover, uncertainty in cost should be accounted for in project planning because activities are exposed to risks that can cause delays and budget overruns. This paper presents a fuzzy-multi-mode resource-constrained discrete-time-cost-resource optimization (F-MRC-DTCRO) model for the time-cost-resource moment tradeoff in a fuzzy environment while satisfying all the project constraints. In the proposed model, fuzzy numbers are used to characterize the uncertainty of direct cost of activities. Using this model, different risk acceptance levels of the decision maker can be addressed in the optimization process. A newly developed multi-objective optimization algorithm called ENSCBO is used to search non-dominated solutions to the fuzzy multi-objective model. Finally, the developed model is applied to solve a benchmark test problem. The results indicate that incorporating the fuzzy structure of uncertainty in costs to previously developed MRC-DTCRO models facilitates the decision-making process and provides more realistic solutions.
\end{abstract}

\section{Keywords}

discrete time-cost tradeoff, resource-constrained project scheduling, resource leveling, optimization, uncertainties, fuzzy theory, ENSCBO, construction management

\section{Introduction}

The role of project management is to make use of knowledge, skills, tools, and techniques to fulfill the project requirements [1]. Construction projects involve a network of activities having a precedence relationship between them, each of which can be completed in a variety of ways. Depending on the adopted construction method, the employed resources, the consumed materials, a particular activity might have a number of alternatives, each with a different completion time, completion cost, and other performance factors. Furthermore, a single execution mode should be used for every activity. Thus, it is crucial to assign the appropriate execution mode to each activity. Time-cost tradeoff problems (TCTP) seek to minimize project costs keeping project duration within desired limits [2]. In general, the cost of accelerating an activity is higher because more expensive resources are usually needed. Therefore, the optimal combination of time and cost to accomplish each activity must be chosen by construction firms. However, it is more practical to use the discrete version of TCTP (DTCTP) in situations where there is a discrete, non-increasing relationship between the number of nonrenewable resources consumed by a project activity and the time it takes to complete [3].

On the other hand, a project's schedule is affected by its resource constraints since executing each activity requires various renewable and nonrenewable resources, that in most cases, they are limited. The limited number of labor, equipment, and amount of materials are examples of resource constraints. The resource-constrained project scheduling problem (RCPSP) method aims to select optimal precedence of activities to minimize project make-span 
by considering precedence relationship constraints and resource constraints. Multi-mode RCPSP (MRCPSP) is a generalized form of RCPSP, in which various execution modes are available for each activity with corresponding resource requirements and duration [4]. In addition, resource planning strategies play an essential role in project success. Excessive variations in resource usage throughout the project duration lead to reduced labor productivity and increased cost and time. Resource management in construction projects is usually handled by solving resource allocation or resource leveling problems (RLP) [5]. Consequently, an efficient construction project schedule can be achieved through a combination of DTCT, MRCPSP, and RLP as a multi-objective optimization problem.

Due to linguistic terms and subjectivity of managers and engineers, the measured performance factors for each project activity, such as time, cost, quality, etc., are generally vague, uncertain, or imprecise. Thus, performance measurements for the overall project are subject to uncertainties [6]. Also, projects often face opportunities and threats that may affect the project's objectives in uncertain environments. Various types of project complexity and the involvement of more parties in contracts make the construction industry and construction projects risky. It is possible to reduce this level of risk by implementing risk management practices. Along with assessing and controlling the schedule for projects, project managers need to manage risks as well $[7,8]$. The outcome of a risk event can differ in favorableness from the most likely outcome and can fall within a certain range, as well [9]. As a means of addressing uncertainties in the scheduling process, fuzzy logic has been applied to construction process modeling and decision-making. An activity's cost and duration are generally assumed to be deterministic. However, in practice, they are uncertain and may be defined as fuzzy numbers. Therefore, considering uncertainties is necessary for any multi-objective scheduling problem (MOSP) when optimizing time and cost, quality, safety, etc. Such a problem is called stochastic MOSP [10].

Recently, metaheuristic optimization algorithms have attracted much attention for applying to real-world problems. Such techniques explore the search space effectively without requiring time-consuming derivative information in order to find global or quasi-global solutions [11]. Metaheuristics with various characteristics are developed and applied throughout various fields, including structural design [12], project scheduling [13], site layout design [14], etc. Biological evolution, social behavior of animals, and physical phenomena are some sources of inspiration for searching in metaheuristics, e.g., genetic algorithm (GA) [15], particle swarm optimization (PSO) [16], colliding bodies optimization (CBO) [17], etc. Many techniques have been developed to solve construction schedule optimization (CSO), divided into mathematical, heuristic, and metaheuristic. Despite guaranteeing optimality, the first group can be time-consuming and rely on gradient information of the objective function [18]. Furthermore, heuristic methods are inefficient in multi-objective problems. Their major problem is that they do not provide decision-makers with enough options to choose the solution that best suits their needs. These issues have been addressed by developing metaheuristic methods to solve multi-objective problems. Metaheuristic methods are not guaranteed to provide optimal solutions, but they have proven their efficiency in finding good solutions that are relative rather than exact [19]. In recent years, many multi-objective evolutionary algorithms (MOEAs) have been proposed, such as non-dominated sorting genetic algorithm (NSGA-II) [20], strength Pareto evolutionary algorithm (SPEA2) [21], Pareto archived evolution strategy (PAES) [22], multi-objective particle swarm optimization (MOPSO) [23], and multi-objective vibrating particles system (MOVPS) [24]. In literature, various MOEAs have been employed to solve the DTCTP, MRCPSP, and RLP.

Zheng et al. [25] proposed a model for time-cost optimization using a GA-based multi-objective approach supported by an adaptive weight approach. Afshar et al. [26] employed multi colony ant principles to develop non-dominated archiving ant colony optimization (NA-ACO) to solve the time-cost optimization problems. To solve the MRCPSPs, Sebt et al. [4] suggested a hybrid genetic algorithm-fully informed particle swarm algorithm (HGFA). In their analysis, the HGFA proved to be one of the most effective approaches in solving the MRCPSP. El-Rayes and Jun [27] utilized a GA-based model to minimize resource fluctuation and resource peak demand at the same time. In addition, they introduced two new metrics for resource-leveling. Ghoddousi et al. [28] developed MRC-DTCRO based on NSGA-II. According to their model, time, cost, and resource moment deviation are minimized concurrently. Fuzzy sets theory has been applied to different types of CSOs to model uncertainty in the activities' time, cost, and other performance factors. Zheng and Ng [29] developed a model in which fuzzy sets theory was applied to predict the time and cost for alternatives of activity considering managers' behavior. Eshtehardian et al. [10] proposed 
a fuzzy representation of uncertainties incorporating into the time-cost tradeoff (TCT) model to evaluate alternatives' direct costs. As a means of incorporating managers' behavior in the process of forecasting time and cost of activities, Zahraie and Tavakolan [30] utilized fuzzy numbers. They proposed an NSGA-II based model to optimize the total time, direct and indirect costs of the project, and the moments of resources concurrently. Kaveh et al. [31] developed a fuzzy resource-constrained project scheduling problem (FRCPSP) model that considers uncertainties in RCPSP utilizing fuzzy numbers for activitys' duration, via two metaheuristics named charged system search (CSS) and colliding bodies optimization (CBO). There have been many studies on nondeterministic CSOs in the literature, but very few have addressed uncertainties in the costs of activities in the MRC-DTCRO model. In this paper, a fuzzy-multimode discrete-time-cost-resource optimization (F-MRCDTCRO) is developed that simultaneously considers MRCDTCRO, risks, and uncertainties. For this purpose, a newly developed MOEA, called enhanced non-dominated sorting colliding bodies optimization (ENSCBO), is employed.

\section{Multi-mode resource-constrained discrete-time-cost- resource optimization (MRC-DTCRO)}

The aim of MRC-DTCRO is to address the problem of scheduling $j=1, \ldots, J$ activities that can be illustrated by an activity-on-node (AON) network, $G=(V . E)$ where nodes and arcs represent the activity set, $V$ and their precedence relationship (without a time lag), $E$, respectively. The set of $\mathcal{M}=\left\{1, \ldots, M_{j}\right\}$ is used to show the available options for performing each activity $j \in V$. In order to execute activity $j$ in mode $m \in \mathcal{M}_{j}, r_{j m k}$ units of renewable resource $k$ must be provided for each period of implementation. $c_{j m}$ and $d_{j m}$ are the direct cost and duration of execution activity $j$ in mode $m$, respectively. It is assumed that after implementing an activity $j$ in mode $m$, that activity cannot be interrupted and its mode cannot be altered, and the progress of activity must be maintained through $d_{j m}$ successive periods. Furthermore, there is limited amount of renewable resources $k=\{1, \ldots, K\}$, available each period and is determined by $R_{k}$. A set of non-dominated solutions for project managers is offered by MRC-DTCRO while minimizing time, cost, and resource moment deviation, given the precedence and resource constraints.

\subsection{Objective functions}

A determination will be made of the duration, direct costs, and resources required for each activity once the mode of execution is chosen. Then a feasible schedule will be generated based on these constraints by incorporating the activity mode information into the schedule generation scheme (SGS). Finally, the project duration, cost, and resource moment can be determined as outputs of the schedule.

Project completion time: Evaluation of a project's success is highly dependent on the project's duration. The first objective of MRC-DTCRO is to minimize the project completion time, which is determined through the SGS. The given schedule indicates when the last activity in a project will be completed, estimating its duration. Therefore the project completion time $F_{t}$ is equal to:

$F_{t}=\max f_{j} . \quad j=1, \ldots, J$,

where $f_{j}$ is the finish time of the $j$ th activity.

Project completion cost: MRC-DTCRO's second objective is to reduce the total project cost. In this model, both the project's direct cost and indirect cost are taken into account. Direct costs refer to the sum of the execution costs for all the activities involved in a project, based on the alternatives chosen for each activity. The indirect cost is deemed constant in each period, and its amount for the entire project changes with project duration. Hence the project completion cost $F_{c}$ can be formulated as follow:

$$
\begin{gathered}
F_{c}=\sum_{j} \sum_{m \in \mathcal{M}_{j}}\left(x_{j m} \times c_{j m}\right)+f_{J} \times c_{i}+y_{J} \times c_{p} \times\left(f_{J}-T_{\text {contract }}\right) . \\
\text { The first term }\left(\sum_{j} \sum_{m \in \mathcal{M}_{j}}\left(x_{j m} \times c_{j m}\right)\right) \text { and second term }\left(f_{J} \times c_{i}\right)
\end{gathered}
$$

of this formula is the total direct and indirect cost of the project, respectively.

Where, $c_{j m}$ is the direct cost of $j$ th activity in mode $m$, and $x_{j m}$ is a decision variable that is defined as follow:

$x_{j m}=\left\{\begin{array}{l}1 \quad \text { if activity } j \text { is performed in mode } m \\ 0 \text { otherwise }\end{array}\right.$.

Throughout a project's duration, $c_{i}$ corresponds to the indirect costs per period.

The contractor will be penalized in case of a delay from the contracted timeline. The term $\left(y_{J} \times c_{p} \times\left(f_{J}-T_{\text {contract }}\right)\right)$ is the penalty cost where $T_{\text {contract }}$ refers to the deadline that is stipulated in the project contract, $c_{p}$ is a penalty in each period of delay, and $y_{J}$ also is the other decision variable that given by:

$y_{J}=\left\{\begin{array}{ll}1 & f_{J}>T_{\text {contract }} \\ 0 & f_{J} \leq T_{\text {contract }}\end{array}\right.$. 
Total resource moment: This model also aims to minimize resource fluctuations throughout the project lifetime considering the deviation of the $X$ moment $M_{x}^{d e v}$ ( $X$ is the time axis) of the resource histogram in the resource leveling process. Resource leveling problem (RLP) is formulated as follows:

$$
M_{x}^{d e v}=\sum_{k=1}^{K} \sum_{t=1}^{T}\left(r_{k}(t)-\bar{r}_{k}\right)^{2},
$$

where $r_{k}(t)$ is the resource usage of renewable resource $k$ in period $t \in\{1, \ldots, T\}$ for a determined schedule, $K$ is the total types of project resources, $T$ is the completion time of project and $\bar{r}_{k}$ is the average resource usage that defined as:

$$
\bar{r}_{k}=\frac{1}{T} \sum_{t=1}^{T} r_{k}(t) \text {. }
$$

\section{Fuzzy logic}

In the fuzzy set (FS) theory, uncertainties lacking a statistical basis are explicitly addressed [32]. Many linguistic descriptions problems can be solved using FS in the real world. [33]. Since construction projects are notoriously imprecise and unpredictable, FS has been extensively used to account for them [34]. From fuzzy set theory, fuzzy logic is derived to deal with a set of membership functions to indicate how much an element belongs to a set, rated from zero (no membership) to one (full membership), and it may also belong to more than one set. Fuzzy logic will become more useful when historical data is scarce or when estimates are not detailed.
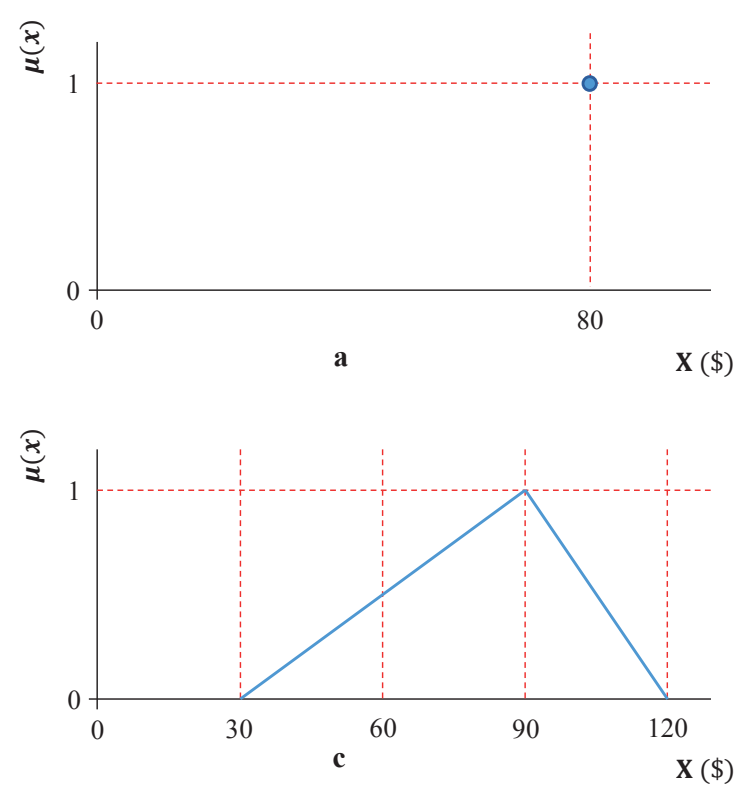

Consider $A$ as a fuzzy number, that is, a normalized convex fuzzy subset of real number $C$ :

$A=\left\{\left(c . \mu_{A}(c)\right) \mid c \in C\right\}$,

where $\mu_{A}(c)$ is a membership function that takes values from Indicating to what degree belongs to $A$. Fuzzy logic uses fuzzy numbers that have a specific distribution [25]. Several fuzzy systems with a single, rectangular, trapezoidal, triangular number or other types have been introduced, as shown in Fig. 1 [35]. The stochastic nature of the parameters of the problem strongly influences the choice of fuzzy number shapes.

In practice, project activity's costs are uncertain due to the influence of many uncontrollable factors, so the assumption of fixed and known costs cannot be justified. In fuzzy scheduling, in which uncertainty is taken into account, fuzzy numbers are used to model activity costs. Using triangular fuzzy numbers is a common method for representing the costs of an activity [29]. Additionally, various operations can be performed on fuzzy numbers, such as unions, intersections, etc. One such operation is the $\alpha$ cut, which ties together fuzzy and crisp sets and functions as the basis for many existing systems. The $\alpha$ cut level set of $A$ can be defined as:

$$
A^{\alpha}=\left\{\left(c . \mu_{A}(c)\right) \geq \alpha \mid c \in C\right\} \quad \forall \alpha \in[0.1]
$$

It is possible to transform fuzzy numbers representing uncertain variables into crisp sets using the $\alpha$ cut concept. In this way, the proposed framework can be utilized to determine optimum options with different alpha cut
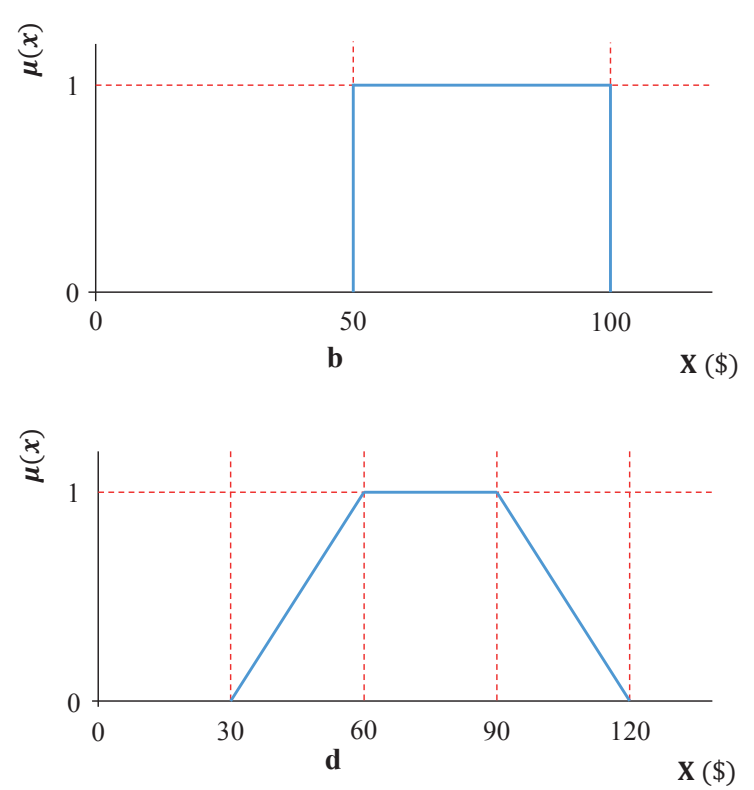

Fig. 1 Different types of fuzzy numbers: a) single value; b) rectangular distribution; c) triangular distribution; d) trapezoidal distribution adapted from [31] 
values, reflecting risk tolerance. Because the value of $\alpha$ can significantly impact non-dominated solutions, decisionmakers must carefully consider its choice.

\section{Metaheuristic algorithm}

MRC-DTCRO's principal goal is to reduce project duration, cost, and resource moment while addressing the priority relationships among activities and restricted resources. Problems of this type are considered NP-hard. Consequently, the exact methods cannot locate Pareto optimal solutions within the logical timeframe. Such problems can be tackled through metaheuristic algorithms. A new type of colliding bodies optimization ( $\mathrm{CBO}$ ), which has recently been adapted to a multi-objective configuration, is used in this research. This section discussed the standard CBO [17], enhanced CBO (ECBO) [36], and multi-objective version of this algorithm, which is called enhanced non-dominated sorting colliding bodies optimization (ENSCBO) [37].

\subsection{Colliding bodies optimization (CBO)}

$\mathrm{CBO}$ is characterized as a physics-based meta-heuristic algorithm that relies on analyzing collisions between bodies in one dimension. Momentum and energy are laws of physics that explain collisions among objects. Whenever objects collide in an isolating system, their total momentum is conserved. Physics conservation laws for colliding bodies have been used to justify the formulation of $\mathrm{CBO}$. CBO's methodology is straightforward: Its best solutions are not stored in memory, nor does it have an internal factor. The following section explains the laws and theories of the algorithm. All of the explanations about this method are taken from [17].

\subsection{CBO formulation}

$\mathrm{CBO}$ employs several agents that represent candidate solutions, such as agent $X_{i}$, which comprises a number of variables (i.e., $X_{i}=\left\{\left(X_{i, j}\right)\right\}$ ) and is defined as a colliding body (CB) with a specific mass. Two types of object groups, including stationary and moving objects, mimic the process of collision. A pair-by-pair collision process occurs during this process, in which moving objects move after stationary objects, improving their positions and making stationary objects move towards more promising spaces. As a result of the collision, each CB is repositioned according to the changes in velocity. Following is a brief outline of the CBO process:

Step 1. Initialization: Initially, $\mathrm{CBs}$ are positioned in the search space using randomly generated individuals: $x_{i}^{0}=x_{\text {min }}+\operatorname{rand}\left(x_{\max }-x_{\text {min }}\right), i=1, \ldots, n$,

where, $x_{i}^{0}$ indicates the initial value vector of the $i$ th CB. $x_{\min }$ and $x_{\max }$ are the lower and the upper bounds of variables, respectively; rand is a random number in the range of [0.1], and $n$ is the number of CBs.

Step 2. Calculating mass: For each CB, the magnitude of the body mass is as follows:

$m_{k}=\frac{\frac{1}{f i t(k)}}{\sum_{i=1}^{n} \frac{1}{f i t(i)}} . k=1, \ldots, n$,

where fit $(i)$ denotes the objective function value of the $i$ th agent while better-performing CBs have a higher mass than their inferior counterparts.

Step 3. Forming groups: First, all CBs are sorted ascendingly according to their objective function values. Then $\mathrm{CBs}$ are categorized into two distinct subgroups: stationary $\mathrm{CBs}$ (the lower half) and moving CBs (the upper half).

Step 4. Pre-collision criteria: The stationary CBs are good agents that have zero velocity before colliding. Each moving $\mathrm{CB}$ moves toward its matching stationary $\mathrm{CB}$, and a collision happens between pairs of CBs. Therefore, the stationary and moving $\mathrm{CBs}$ have the following initial velocities:

$v_{i}=0 . \quad i=1, \ldots, \frac{n}{2}$,
$v_{i}=x_{i}-x_{i-\frac{n}{2}} . \quad i=\frac{n}{2}+1, \ldots, n$,

where $v_{i}$ and $x_{i}$ are the velocity and position of the $i$ th CB, respectively.

Step 5. Post-collision criteria: The velocity of each stationary $\mathrm{CB}$ after the collision is determined as follow:

$v_{i}^{\prime}=\frac{\left(m_{i+\frac{n}{2}}+\varepsilon m_{i+\frac{n}{2}}\right) v_{i+\frac{n}{2}}}{m_{i}+m_{i+\frac{n}{2}}} . \quad i=1, \ldots, \frac{n}{2}$,

where, $v_{i+\frac{n}{2}}$ and $v_{i}{ }^{\prime}$ are the velocity of the $i$ th moving $\mathrm{CB}$ before and the $i$ th stationary $\mathrm{CB}$ after the collision, respectively, $m_{i}$ is the mass of the $i$ th $\mathrm{CB}$, and $m_{i+\frac{n}{2}}$ is the mass of
the $i$ th moving $\mathrm{CB}$ pair. The velocity of $i$ th moving $\mathrm{CB}$ after the collision is given by: 
$v_{i}^{\prime}=\frac{\left(m_{i}-\varepsilon m_{i-\frac{n}{2}}\right) v_{i}}{m_{i}+m_{i-\frac{n}{2}}} . \quad i=\frac{n}{2}+1, \ldots, n$,

where, $v_{i}$ and $v_{i}{ }^{\prime}$ are the velocity of the th moving $\mathrm{CB}$ before and after the collision, respectively, $m_{i}$ is the mass of the $i$ th $\mathrm{CB}$, and $m_{i-\frac{n}{2}}$ is the mass of the $i$ th CB pair.

The factor of $\varepsilon$ is the coefficient of restitution (COR) that decreases from 1 to 0 on a linear basis. So, it is defined as:

$$
\varepsilon=1-\frac{\text { iter }}{\text { iter }_{\max }},
$$

where iter and iter $_{\text {max }}$ are the number of the current iteration and the total number of iterations, respectively

Step 6. Generating new CBs: CBs are repositioned after they collide based on the corresponding velocity and the locations of stationary CBs.

The new position of each stationary $\mathrm{CB}$ is:

$$
x_{i}^{\text {new }}=x_{i}+\operatorname{rand} \circ v_{i}^{\prime} . \quad i=1, \ldots, \frac{n}{2},
$$

where $x_{i}^{n e w}, x_{i}$ and $v_{i}^{\prime}$ are the new position, old position, and the velocity of the $i$ th stationary CB after the collision, respectively. rand is a random vector uniformly distributed in the [-1.1] interval, and the sign "०" denotes an element-by-element multiplication.

Also, the new positions of moving CBs are obtained by:

$$
x_{i}^{\text {new }}=x_{i-\frac{n}{2}}+\operatorname{rand} \circ v_{i}^{\prime} \quad i=\frac{n}{2}+1, \ldots, n,
$$

where, $x_{i}^{\text {new }}$ and $v_{i}{ }^{\prime}$ are the new position and the velocity after the collision of the $i$ th moving $\mathrm{CB}$, respectively, $x_{i-\frac{n}{2}}$ is the previous position of the stationary CB pair.

Step 6. Terminal condition: The optimization process is finished when the determined stopping criteria are met. Otherwise, go to Step 2 for a new iteration.

\subsection{Enhanced colliding bodies optimization (ECBO)}

ECBO is an updated version of the standard $\mathrm{CBO}$ since it has been improved in terms of both the quality of the solutions and convergence speed by Kaveh and Ilchi Ghazaan [36]. It was modified to keep previous best solutions in memory and prevent the algorithm from trapping in local optima. In the latter mechanism, one component of each CB is selected at random and is altered with a certain probability (which is defined by parameter Pro) as follow: $x_{i j}=x_{j . \min }+\operatorname{random} \cdot\left(x_{j . \max }-x_{j . \min }\right)$,

where $x_{i j}$ is the $j$ th variable of the $i$ th CB. $x_{j . \min }$ and $x_{j . \max }$ are the lower and upper bounds of the $j$ th variable, respectively.

\subsection{Enhanced non-dominated sorting colliding bodies optimization (ENSCBO)}

As standard $\mathrm{CBO}$ and $\mathrm{ECBO}$ are originally single-objective approaches, they are not helpful in solving problems consisting of more than one objective function. Kaveh et al [37] adapted the configuration of ECBO to handle the multi-objective optimization problems by employing a non-dominated sorting technique represented by Deb et al. [20] and proposed a new algorithm named ENSCBO. The CBs are divided into separate fronts using this method, and the number of a CB's front determines its ranking. The crowding distance (CD), another concept in NSGA-II [20], is used to determine the priority of CBs in each front. In this way, CD prioritizes solitude solutions above others in the same front to maintain the diversity of solutions. For each solution, crowding distance is formulated as:

$C D^{i}=\sum_{j=1}^{k} \frac{\left|f_{j}^{i+1}-f_{j}^{i-1}\right|}{f_{j}^{\max }-f_{j}^{\min }} . \quad j=1, \ldots, k$,

where $f_{j}^{i+1}$ and $f_{j}^{i-1}$ are the $j$ th function value of the $(i+1)$ th and $(i-1)$ th $\mathrm{CB}$ in the front, respectively. Furthermore, $f_{j}^{\max }$ and $f_{j}^{\min }$ are the maximum and minimum values of the $j$ th objective function, respectively. In this algorithm, the magnitude of the mass for each $\mathrm{CB}$ is calculated using the rank and $\mathrm{CD}$ values of the $\mathrm{CBs}$ as follow:

$$
m_{k}=\frac{\frac{1}{\operatorname{Rank}(k)+\frac{1}{C D(k)}}}{\sum_{i=1}^{n} \frac{1}{\operatorname{Rank}(i)+\frac{1}{C D(i)}}} \quad k=1 \ldots \ldots n,
$$

The rest of the steps and details are the same as those used by the ECBO.

\section{Fuzzy-multi-mode resource-constrained discrete- time-cost-resource optimization (F-MRC-DTCRO)} In the proposed F-MRC-DTCRO, factors such as duration, amount of resource usage, and fuzzy numbers of costs for each alternative are defined as inputs to the optimization algorithm. In the same way as real numbers, fuzzy numbers can be manipulated by using extension principles. 
For a given candidate solution, one of the objectives is to determine the total project cost. At first, this can be done by calculating the fuzzy total costs of a set of selected modes as follow: Consider $\tilde{C}$ as a fuzzy number representing the performance cost of an execution mode, so its $\alpha$ cut can be denoted as follows:

$$
C_{\alpha}=\left[c_{\alpha}^{-} \cdot c_{\alpha}^{+}\right] \text {, }
$$

where according to a certain value of $\alpha, c_{\alpha}^{-}$and $c_{\alpha}^{+}$represent the lower and upper limits of the fuzzy cost, respectively, as shown in Fig. 2.

Additionally, for two fuzzy costs, $\tilde{C}_{1}$ and $\tilde{C}_{2}$ and their $\alpha$ cuts, $C 1_{\alpha}$ and $C 2_{\alpha}$, the summation of them can be defined as [38]:

$$
(C 1+C 2)_{\alpha}=\left[c 1_{\alpha}^{-}+c 2_{\alpha}^{-} \cdot c 1_{\alpha}^{+}+c 2_{\alpha}^{+}\right] .
$$

The above-mentioned formulation can be extended to encompass all fuzzy costs of selected options for activities, resulting in a single fuzzy number that represents the project's total cost.

Moreover, to compare the candidate solutions in terms of total project cost, associated fuzzy costs for different $\alpha$ cut values should be ranked. In order to convert the total fuzzy cost to a crisp value, a defuzzification method is applied. Based on this technique, consider the total fuzzy cost, $\tilde{C}$ with membership function, $A$, in the case of a candidate solution, the area captured by $A$ is defined by point $C^{*}$ based on the center of gravity defuzzifier. Thus, $C^{*}$ stands for the total cost crisp value and is calculated as follows:

$$
C^{*}=\frac{\int C \mu_{A}(c) d c}{\int \mu_{A}(c) d c} .
$$

As a result, it would be possible to compare the candidate solutions based on the associated value of $C^{*}$ and two other objective functions. Flowchart of the proposed model of F-MRC-DTCRO is explained in Fig. 3.

\section{Model application and discussion of the results}

To verify and demonstrate the application of the proposed F-MRC-DTCRO model using ENSCBO, an exciting case study of a warehouse construction project, which was firstly introduced by Chen and Weng [39], is chosen. Ghoddousi et al. [28] made some modifications to the project activities data to solve the MRC-DTCRO problem in a certain environment. This case study presents a project consisting of 37 activities, each involving multiple execution methods. A single type of renewable resource is available, with a daily limit of 12 workers. Also, indirect costs are considered to be zero during the project timeline. In this study, non-symmetric triangle shapes are assumed to represent the cost of the alternatives. These cost values are transformed into three numbers, of which the first, middle, and third are the minimum, most probable, and maximum cost of the assigned fuzzy number. Details of this case study and the highest and lowest possible costs of each available alternative are presented in Table 1 . The network of the case

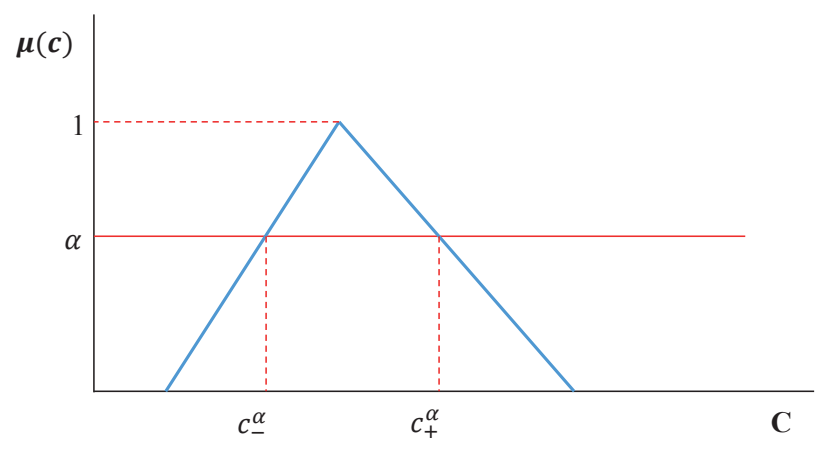

Fig. $2 \alpha$ cut of a fuzzy cost

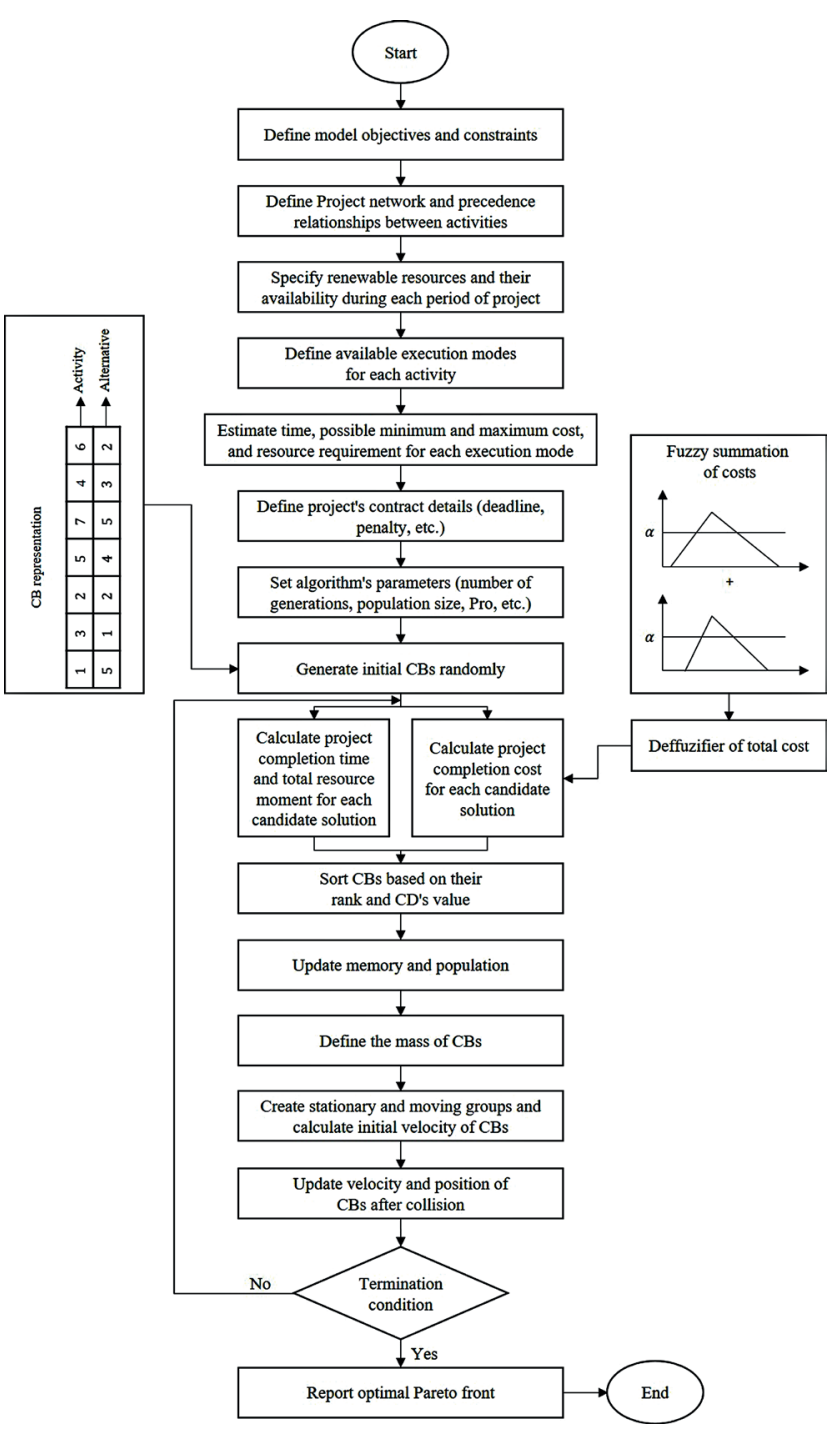

Fig. 3 Flowchart of proposed F-MRC-DTCRO model 
Table 1 Activity data of the case study

\begin{tabular}{|c|c|c|c|c|c|c|c|c|}
\hline $\begin{array}{l}\text { Act. } \\
\text { ID }\end{array}$ & Act. description & Execution & Duration & Predecessor & Labor requirement & \multicolumn{3}{|c|}{ Direct cost (\$) } \\
\hline 1 & Mobilization and site facilities & 1 & 25 & - & 2 & 4800 & 5000 & 5600 \\
\hline 2 & Soil test & 1 & 11 & - & 2 & 1900 & 2200 & 2700 \\
\hline \multirow[t]{2}{*}{3} & Excavation work & 1 & 21 & 1 & 4 & 8000 & 8400 & 9100 \\
\hline & & 2 & 16 & 1 & 6 & 9500 & 9600 & 10000 \\
\hline \multirow[t]{2}{*}{4} & Piling work & 1 & 20 & 1 & 5 & 9400 & 10000 & 12500 \\
\hline & & 2 & 18 & 1 & 6 & 9750 & 10800 & 13000 \\
\hline 5 & Pile loading test & 1 & 15 & 2 & 2 & 2800 & 3000 & 3500 \\
\hline \multirow[t]{2}{*}{6} & Backfilling and M\&E work & 1 & 9 & 4 & 3 & 2550 & 2700 & 3400 \\
\hline & & 2 & 6 & 4 & 5 & 2600 & 3000 & 3600 \\
\hline \multirow[t]{2}{*}{7} & Pile cap work & 1 & 14 & 2,4 & 4 & 5500 & 5600 & 6100 \\
\hline & & 2 & 10 & 2,4 & 6 & 5700 & 6000 & 6550 \\
\hline 8 & Column rebar and M\&E work & 1 & 10 & 5 & 5 & 4850 & 5000 & 5300 \\
\hline \multirow[t]{2}{*}{9} & Slab casting & 1 & 12 & $3,6,7$ & 5 & 5800 & 6000 & 6700 \\
\hline & & 2 & 11 & $3,6,7$ & 6 & 6400 & 6600 & 7200 \\
\hline 10 & Column formwork & 1 & 10 & 8 & 4 & 3850 & 4000 & 4300 \\
\hline 11 & Roof beam and slab formwork & 1 & 12 & 9 & 5 & 5800 & 6000 & 6400 \\
\hline 12 & Column casting & 1 & 10 & 10 & 4 & 3700 & 4000 & 4900 \\
\hline 13 & Roof beam and slab rebar & 1 & 10 & 11,12 & 5 & 4800 & 5000 & 5450 \\
\hline 14 & Roof parapet wall casting & 1 & 14 & 12 & 5 & 6600 & 7000 & 7800 \\
\hline 15 & M\&E work 1 & 1 & 7 & 12 & 4 & 2750 & 2800 & 3100 \\
\hline 16 & Door and window frame & 1 & 7 & 14 & 3 & 2000 & 2100 & 2500 \\
\hline 17 & M\&E work 2 & 1 & 7 & 13,14 & 4 & 2650 & 2800 & 3300 \\
\hline \multirow[t]{2}{*}{18} & Roof slab casting & 1 & 12 & 15 & 4 & 4500 & 4800 & 5500 \\
\hline & & 2 & 9 & 15 & 6 & 5250 & 5400 & 6000 \\
\hline 19 & Plastering work & 1 & 10 & 16,17 & 4 & 3800 & 4000 & 4400 \\
\hline \multirow[t]{2}{*}{20} & Brick wall laying & 1 & 14 & 18 & 4 & 5400 & 5600 & 6200 \\
\hline & & 2 & 10 & 18 & 6 & 5650 & 6000 & 6700 \\
\hline \multirow[t]{2}{*}{21} & Ceiling skimming & 1 & 7 & 11 & 4 & 2700 & 2800 & 3150 \\
\hline & & 2 & 14 & 20 & 3 & 4000 & 4200 & 4500 \\
\hline 22 & Toilet floor and wall tiling work & 1 & 10 & 20 & 5 & 4600 & 5000 & 5700 \\
\hline 23 & Drain work & 1 & 10 & 19,21 & 4 & 3850 & 4000 & 4350 \\
\hline 24 & Apron slab casting & 1 & 9 & 21,23 & 5 & 4400 & 4500 & 4900 \\
\hline 25 & Door and window & 1 & 7 & 22 & 5 & 3400 & 3500 & 3800 \\
\hline 26 & Painting work & 1 & 14 & 19,22 & 4 & 5400 & 5600 & 6100 \\
\hline 27 & Fencing work & 1 & 16 & 24 & 5 & 7500 & 8000 & 8800 \\
\hline \multirow[t]{2}{*}{28} & External wall plastering & 1 & 10 & 25,26 & 4 & 3800 & 4000 & 4600 \\
\hline & & 2 & 9 & 25,26 & 5 & 4200 & 4500 & 5300 \\
\hline 29 & Electrical final fix & 1 & 6 & 25 & 2 & 1100 & 1200 & 1500 \\
\hline 30 & Main gate installation & 1 & 3 & 24,27 & 3 & 850 & 900 & 1100 \\
\hline 31 & External wall painting & 1 & 12 & 29 & 4 & 4600 & 4800 & 5300 \\
\hline 32 & Qualified person inspection & 1 & 5 & 27,30 & 2 & 950 & 1000 & 1150 \\
\hline 33 & Landscape work & 1 & 10 & 28,31 & 2 & 1900 & 2000 & 2300 \\
\hline 34 & Registered inspector inspection & 1 & 7 & 32,33 & 1 & 650 & 700 & 800 \\
\hline 35 & Authority inspection & 1 & 7 & 34 & 1 & 650 & 700 & 800 \\
\hline 36 & Defect work & 1 & 14 & 35 & 1 & 1300 & 1400 & 1650 \\
\hline 37 & Project handover & 1 & 1 & 36 & 1 & 70 & 100 & 150 \\
\hline
\end{tabular}


study is shown in Fig. 4. The objective of this case is to find non-dominant solutions with the intention of optimizing project time, cost, and resource deviation. Implementation of the model was done using MATLAB R2018b [40].

An optimal set of 48 unique Pareto solutions satisfying the desired project objectives were found. Project completion time, project completion cost, and total resource moment were determined for every 48 implementation scenarios of project. The time, the fuzzy costs for different $\alpha$ cuts and resource moment for all 48 obtained Pareto optimal solutions are presented in Table 2. Fig. 5 shows the results of the algorithm after 200 iterations of 50 agents for different $\alpha$ values. It should be noted that the best performance of ENSCBO in terms of performance metrics of multi-objective algorithms such as number of Pareto solutions and diversification metric is determined by trials and errors. Project completion time values vary from 190 to 231 days, project completion cost values for $\alpha=1$ vary from $\$ 145400$ to $\$ 147700$, total resource moment values vary from 1811.3 to 2721.6. Comparing the proposed model's outputs for $\alpha=1$ with corresponding results of the earlier deterministic version of MRC-DTCRO validated its performance. Once $\alpha=1$, the stochastic nature of the presented problem becomes deterministic, so that comparisons with deterministic models can be made easily. The results of the proposed ENSCBO with $\alpha=1$ are very similar and even superior to those of a similar problem solved by Ghoddousi et al. [28], which is depicted in Table 3. While the average time for ENSCBO (203.31 days) is very slightly different from that of NSGA-II (203.04 days), the average cost and resource moment deviation for ENSCBO (\$145958.33 and 2210.45 ) is less than those of NSGA-II (\$146015.56 and 2222.28). In addition, the proposed model is capable to use a variety of $\alpha$ cut values in the fuzzy cost assessment process, allowing the project planners to choose a suitable value for the $\alpha$ to set the level of risk retention. As shown in Table 2 with increasing $\alpha$ values, the difference between the minimum and maximum expected costs of the project decreases, which signifies that the project manager takes on more risk. The decision to use 1 as $\alpha$ (i.e., 100\% risk acceptance) results in an entirely certain circumstance and makes cost estimation uncertainty invisible. In contrast, with a zero risk acceptance level, 0 may be chosen as the $\alpha$ value, which would result in an extremely wide range of costs. The project manager must know the expected minimum and maximum total costs since the cumulative impact of uncertainties in the cost of alternatives can lead to a wide estimate of the project's total cost. Although this model provides construction planners and decision-makers with a practical tool for project scheduling, it is also possible to include other types of objectives such as safety and quality in the planning process. In this study, other objectives of the project were not considered in the optimization process since project-specific details of the case study were not available.

\section{Conclusions}

In this study, an F-MRC-DTCRO model is presented to address the time-cost-resource moment tradeoff problem considering uncertainties in costs. With ENSCBO, a recently introduced multi-objective optimization algorithm, the proposed framework attempts to minimize the project's time, cost, and resource moment as three objectives. The modeling framework fully incorporates fuzzy sets theory to account for uncertainty in project costs. In order to illustrate how the model can be applied to the

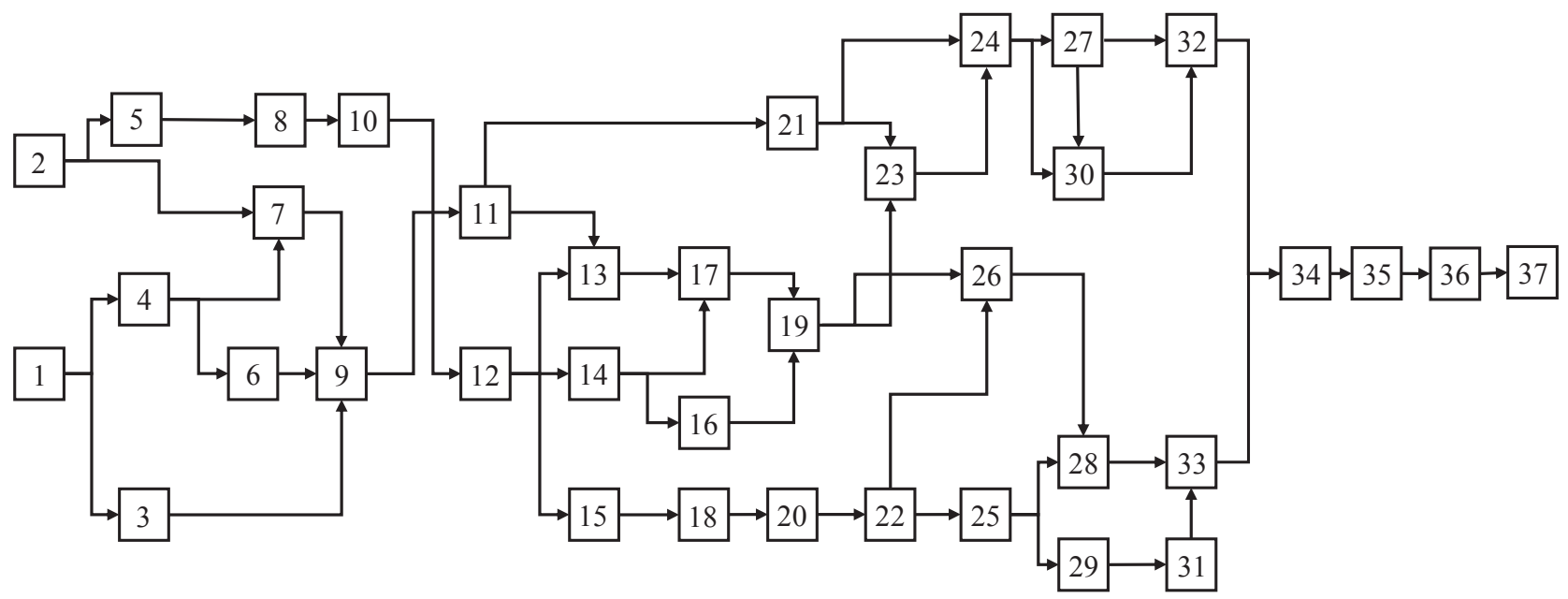

Fig. 4 Project network activities of case study 
Table 2 F-MRC-DTCRO model results for case study

\begin{tabular}{|c|c|c|c|c|c|c|c|c|c|c|c|}
\hline \multirow{3}{*}{$\begin{array}{l}\text { Solution } \\
\text { no. }\end{array}$} & \multirow{3}{*}{$\begin{array}{c}\begin{array}{c}\text { Time } \\
\text { (day) }\end{array} \\
190\end{array}$} & \multicolumn{9}{|c|}{ Cost (\$) } & \multirow{3}{*}{$\begin{array}{r}\begin{array}{r}\text { Resource } \\
\text { moment }\end{array} \\
2719.3\end{array}$} \\
\hline & & \multicolumn{2}{|c|}{$\alpha=0$} & \multicolumn{2}{|c|}{$\alpha=0.25$} & \multicolumn{2}{|c|}{$\alpha=0.5$} & \multicolumn{2}{|c|}{$\alpha=0.75$} & \multirow{2}{*}{$\frac{\alpha=1}{147700}$} & \\
\hline & & 140320 & 165900 & 142172 & 161347 & 144010 & 156800 & 145862 & 152247 & & \\
\hline 2 & 191 & 139920 & 165200 & 141747 & 160697 & 143560 & 156200 & 145387 & 151697 & 147200 & 2721.6 \\
\hline 3 & 192 & 139570 & 164700 & 141284 & 160122 & 142985 & 155550 & 144699 & 150972 & 146400 & 2587.0 \\
\hline 4 & 193 & 139220 & 164900 & 140996 & 160247 & 142760 & 155600 & 144536 & 150947 & 146300 & 2595.0 \\
\hline 5 & 194 & 138820 & 164200 & 140571 & 159597 & 142310 & 155000 & 144061 & 150397 & 145800 & 2592.5 \\
\hline 6 & 194 & 139620 & 164900 & 141396 & 160347 & 143160 & 155800 & 144936 & 151247 & 146700 & 2565.8 \\
\hline 7 & 194 & 139220 & 164900 & 141146 & 160397 & 143060 & 155900 & 144986 & 151397 & 146900 & 2537.5 \\
\hline 8 & 195 & 138820 & 164200 & 140571 & 159597 & 142310 & 155000 & 144061 & 150397 & 145800 & 2552.6 \\
\hline 9 & 195 & 138870 & 164400 & 140683 & 159822 & 142485 & 155250 & 144298 & 150672 & 146100 & 2464.7 \\
\hline 10 & 196 & 138570 & 163700 & 140283 & 159122 & 141985 & 154550 & 143698 & 149972 & 145400 & 2539.7 \\
\hline 11 & 196 & 138820 & 164200 & 140571 & 159597 & 142310 & 155000 & 144061 & 150397 & 145800 & 2472.3 \\
\hline 12 & 196 & 139420 & 164700 & 141171 & 160122 & 142910 & 155550 & 144661 & 150972 & 146400 & 2412.8 \\
\hline 13 & 196 & 140020 & 165850 & 141846 & 161209 & 143660 & 156575 & 145486 & 151934 & 147300 & 2411.0 \\
\hline 14 & 197 & 138570 & 163700 & 140283 & 159122 & 141985 & 154550 & 143698 & 149972 & 145400 & 2526.4 \\
\hline 15 & 197 & 138620 & 163900 & 140395 & 159347 & 142160 & 154800 & 143935 & 150247 & 145700 & 2353.1 \\
\hline 16 & 197 & 139020 & 164650 & 140821 & 160034 & 142610 & 155425 & 144411 & 150809 & 146200 & 2330.0 \\
\hline 17 & 198 & 138570 & 163700 & 140283 & 159122 & 141985 & 154550 & 143698 & 149972 & 145400 & 2422.6 \\
\hline 18 & 198 & 138820 & 164200 & 140571 & 159597 & 142310 & 155000 & 144061 & 150397 & 145800 & 2325.8 \\
\hline 19 & 198 & 138970 & 164400 & 140708 & 159772 & 142435 & 155150 & 144173 & 150522 & 145900 & 2306.1 \\
\hline 20 & 199 & 138570 & 163700 & 140283 & 159122 & 141985 & 154550 & 143698 & 149972 & 145400 & 2298.3 \\
\hline 21 & 199 & 138770 & 164150 & 140533 & 159559 & 142285 & 154975 & 144048 & 150384 & 145800 & 2235.8 \\
\hline 22 & 200 & 138570 & 163700 & 140283 & 159122 & 141985 & 154550 & 143698 & 149972 & 145400 & 2215.4 \\
\hline 23 & 200 & 139070 & 164850 & 140933 & 160259 & 142785 & 155675 & 144648 & 151084 & 146500 & 2213.9 \\
\hline 24 & 201 & 138570 & 163700 & 140283 & 159122 & 141985 & 154550 & 143698 & 149972 & 145400 & 2142.0 \\
\hline 25 & 202 & 138820 & 164350 & 140645 & 159784 & 142460 & 155225 & 144285 & 150659 & 146100 & 2132.1 \\
\hline 26 & 203 & 138870 & 164400 & 140683 & 159822 & 142485 & 155250 & 144298 & 150672 & 146100 & 2114.1 \\
\hline 27 & 204 & 138570 & 163700 & 140283 & 159122 & 141985 & 154550 & 143698 & 149972 & 145400 & 2130.7 \\
\hline 28 & 204 & 138620 & 163900 & 140395 & 159347 & 142160 & 154800 & 143935 & 150247 & 145700 & 2076.9 \\
\hline 29 & 205 & 138570 & 163700 & 140283 & 159122 & 141985 & 154550 & 143698 & 149972 & 145400 & 2065.2 \\
\hline 30 & 205 & 138620 & 163900 & 140395 & 159347 & 142160 & 154800 & 143935 & 150247 & 145700 & 2045.6 \\
\hline 31 & 205 & 138870 & 164400 & 140683 & 159822 & 142485 & 155250 & 144298 & 150672 & 146100 & 2032.7 \\
\hline 32 & 205 & 139220 & 165100 & 141045 & 160447 & 142860 & 155800 & 144685 & 151147 & 146500 & 2023.6 \\
\hline 33 & 206 & 138620 & 163900 & 140395 & 159347 & 142160 & 154800 & 143935 & 150247 & 145700 & 2015.9 \\
\hline 34 & 206 & 139620 & 165100 & 141420 & 160522 & 143210 & 155950 & 145010 & 151372 & 146800 & 2008.7 \\
\hline 35 & 207 & 138570 & 163700 & 140283 & 159122 & 141985 & 154550 & 143698 & 149972 & 145400 & 2062.9 \\
\hline 36 & 207 & 138620 & 163900 & 140395 & 159347 & 142160 & 154800 & 143935 & 150247 & 145700 & 1917.7 \\
\hline 37 & 208 & 138570 & 163700 & 140283 & 159122 & 141985 & 154550 & 143698 & 149972 & 145400 & 2048.0 \\
\hline 38 & 210 & 138620 & 163900 & 140395 & 159347 & 142160 & 154800 & 143935 & 150247 & 145700 & 1894.2 \\
\hline 39 & 211 & 138870 & 164400 & 140683 & 159822 & 142485 & 155250 & 144298 & 150672 & 146100 & 1886.8 \\
\hline 40 & 212 & 138620 & 163900 & 140395 & 159347 & 142160 & 154800 & 143935 & 150247 & 145700 & 1845.6 \\
\hline 41 & 212 & 139220 & 165100 & 141045 & 160447 & 142860 & 155800 & 144685 & 151147 & 146500 & 1811.3 \\
\hline 42 & 213 & 138570 & 163700 & 140283 & 159122 & 141985 & 154550 & 143698 & 149972 & 145400 & 2010.6 \\
\hline 43 & 216 & 138620 & 163900 & 140395 & 159347 & 142160 & 154800 & 143935 & 150247 & 145700 & 1829.0 \\
\hline 44 & 217 & 138570 & 163700 & 140283 & 159122 & 141985 & 154550 & 143698 & 149972 & 145400 & 1991.5 \\
\hline 45 & 218 & 138570 & 163700 & 140283 & 159122 & 141985 & 154550 & 143698 & 149972 & 145400 & 1990.2 \\
\hline 46 & 221 & 138570 & 163700 & 140283 & 159122 & 141985 & 154550 & 143698 & 149972 & 145400 & 1977.9 \\
\hline 47 & 225 & 138620 & 163900 & 140395 & 159347 & 142160 & 154800 & 143935 & 150247 & 145700 & 1826.1 \\
\hline 48 & 231 & 139220 & 164400 & 140995 & 159872 & 142760 & 155350 & 144535 & 150822 & 146300 & 1823.3 \\
\hline
\end{tabular}




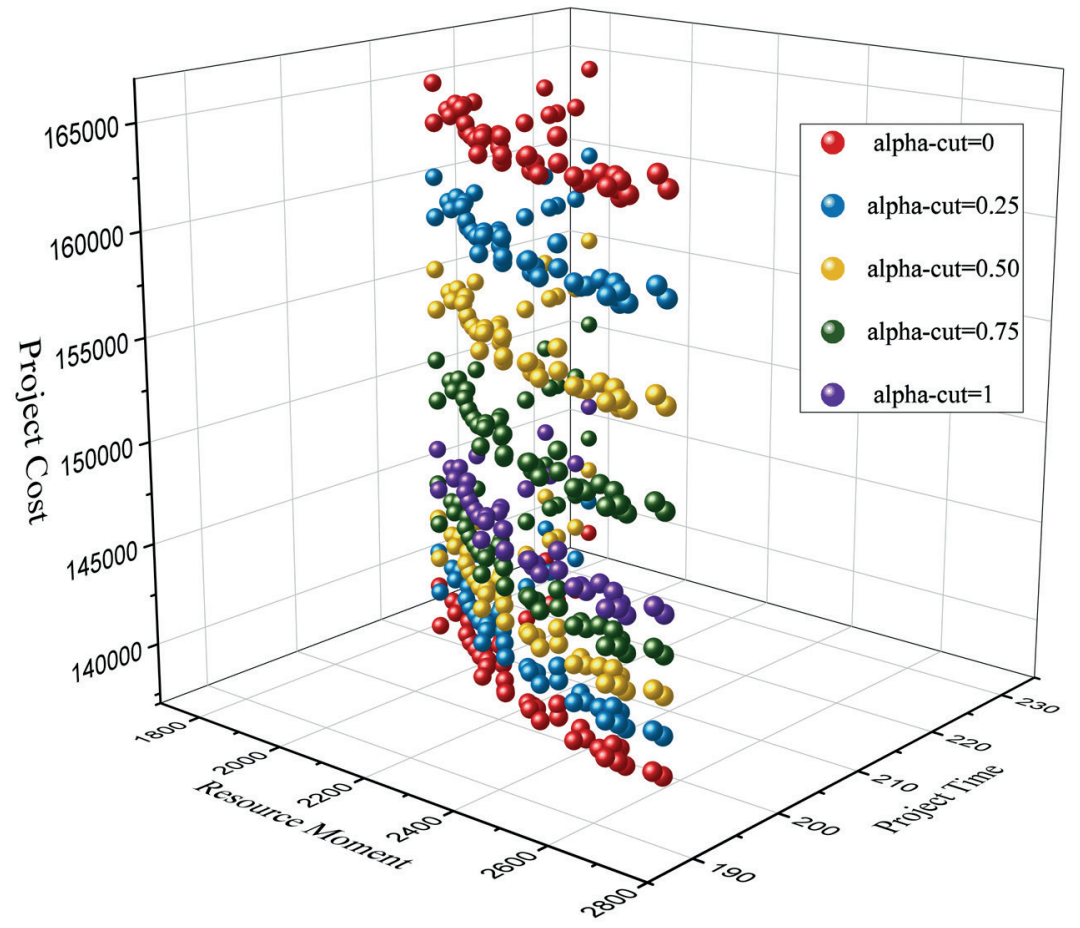

Fig. 5 Fuzzy Pareto fronts associated with different $\alpha$ values

Table 3 Optimal results for certain MRC-DTCRO adapted from Ghoddousi et al. [28]

\begin{tabular}{|c|c|c|c|c|c|c|c|}
\hline Solution no. & Time & Cost & Resource moment & Solution no. & Time & Cost & Resource moment \\
\hline 1 & 190 & 147700 & 2719.27 & 24 & 203 & 145800 & 2170.26 \\
\hline 2 & 191 & 147200 & 2721.58 & 25 & 203 & 146100 & 2118.12 \\
\hline 3 & 192 & 146400 & 2587 & 26 & 204 & 145400 & 2130.69 \\
\hline 4 & 193 & 146300 & 2595.01 & 27 & 204 & 146100 & 2089.66 \\
\hline 5 & 194 & 145800 & 2592.45 & 28 & 204 & 146600 & 2084.92 \\
\hline 6 & 194 & 146700 & 2565.76 & 29 & 205 & 145700 & 2075.64 \\
\hline 7 & 195 & 145800 & 2484.65 & 30 & 205 & 146100 & 2038.7 \\
\hline 8 & 195 & 146100 & 2464.74 & 31 & 205 & 146500 & 2025.61 \\
\hline 9 & 196 & 145400 & 2539.69 & 32 & 206 & 145400 & 2083.3 \\
\hline 10 & 196 & 147600 & 2442.82 & 33 & 206 & 146100 & 2033.25 \\
\hline 11 & 197 & 145400 & 2538.45 & 34 & 207 & 145400 & 2062.88 \\
\hline 12 & 197 & 146100 & 2433.87 & 35 & 207 & 145700 & 1919.69 \\
\hline 13 & 197 & 146200 & 2414.03 & 36 & 210 & 145700 & 1918.19 \\
\hline 14 & 197 & 146600 & 2404.58 & 37 & 211 & 146100 & 1886.79 \\
\hline 15 & 197 & 147000 & 2388.96 & 38 & 212 & 146100 & 1870.5 \\
\hline 16 & 198 & 145700 & 2393.54 & 39 & 213 & 145400 & 2010.57 \\
\hline 17 & 198 & 146200 & 2346.83 & 40 & 213 & 145700 & 1886.57 \\
\hline 18 & 199 & 145400 & 2354.3 & 41 & 213 & 146500 & 1852.83 \\
\hline 19 & 199 & 145800 & 2307.77 & 42 & 217 & 145400 & 1991.53 \\
\hline 20 & 200 & 145400 & 2215.42 & 43 & 217 & 145700 & 1868.29 \\
\hline 21 & 201 & 145400 & 2198.01 & 44 & 226 & 145400 & 1983.5 \\
\hline 22 & 202 & 145800 & 2192.42 & 45 & 226 & 145700 & 1861.86 \\
\hline 23 & 202 & 146100 & 2138.06 & & & & \\
\hline
\end{tabular}


MRC-DTCRO under uncertain conditions, a case study was developed. It was demonstrated that project managers could effectively impose their risk acceptance threshold using the $\alpha$ technique. The effect of different values of $\alpha$ cuts was considered on the estimated total project cost. Based on the results, it is evident that decreasing $\alpha$ value can result in a larger range of total project costs. Comparing the identified non-dominated solutions for $\alpha$ cut $=1$ with those of the previously developed deterministic model proposed by Ghoddousi et al. [28] confirms the efficiency of the proposed ENSCBO in solving the MRC-DTCRO problems. This model can be effectively employed by project managers who have some knowledge

\section{References}

[1] Project Management Institute "A Guide to the Project Management Body of Knowledge PMBOK® Guide", $6^{\text {th }}$ ed., Project Management Institute, Newtown Square, PA, USA, 2017.

[2] Agdas, D., Warne, D. J., Osio-Norgaard, J., Masters, F. J. "Utility of Genetic Algorithms for Solving Large-Scale Construction Time-Cost Trade-Off Problems", Journal of Computing in Civil Engineering, 32(1), Article number 040170721-10, 2018.

https://doi.org/10.1061/(ASCE)CP.1943-5487.0000718

[3] Aminbakhsh, S., Sonmez, R. "Pareto Front Particle Swarm Optimizer for Discrete Time-Cost Trade-Off Problem", Journal of Computing in Civil Engineering, 31(1), Article number 040160401-10, 2017. https://doi.org/10.1061/(ASCE)CP.1943-5487.0000606

[4] Sebt, M. H., Afshar, M. R., Alipouri, Y. "Hybridization of genetic algorithm and fully informed particle swarm for solving the multi-mode resource-constrained project scheduling problem", Engineering Optimization, 49(3), pp. 513-530, 2017. https://doi.org/10.1080/0305215X.2016.1197610

[5] Koulinas, G. K., Anagnostopoulos, K. P. "Construction Resource Allocation and Leveling Using a Threshold Accepting-Based Hyperheuristic Algorithm", Journal of Construction Engineering and Management, 138(7), pp. 854-863, 2012.

https://doi.org/10.1061/(ASCE)CO.1943-7862.0000492

[6] Fayek, A. R. "Fuzzy Logic and Fuzzy Hybrid Techniques for Construction Engineering and Management", Journal of Construction Engineering and Management, 146(7), Article number 04020064112,2020 .

https://doi.org/10.1061/(ASCE)CO.1943-7862.0001854

[7] Nawaz, A., Su, X., Mohi Ud Din, Q., Khalid, M. I., Bilal, M., Shah, S. A. R. "Identification of the H\&S (Health and Safety Factors) Involved in Infrastructure Projects in Developing Countries-A Sequential Mixed Method Approach of OLMT-Project", International Journal of Environmental Research and Public Health, 17(2), Article number 635, 2020.

https://doi.org/10.3390/ijerph17020635

[8] Nawaz, A., Waqar, A., Shah, S. A. R., Sajid, M., Khalid, M. I. "An innovative framework for risk management in construction projects in developing countries: Evidence from Pakistan", Risks, 7(1), Article number 24, 2019.

https://doi.org/10.3390/risks7010024 of risk management basics. As an assumption, no preferences on time, cost, and resource moment were given in the analyzing process. Therefore, if a manager had specific priorities, choosing the preferred scenario among obtained non-dominated solutions would be fairly straightforward. Future studies can enhance the applicability of the model by testing several metaheuristic algorithms. Moreover, the uncertainties in the estimation of time and other project objectives can be considered.

\section{Conflict of interest}

On behalf of all authors, the corresponding author states that there is no conflict of interest.

[9] Eshtehardian, E., Afshar, A., Abbasnia, R. "Fuzzy-based MOGA approach to stochastic time-cost trade-off problem", Automation in Construction, 18(5), pp. 692-701, 2009. https://doi.org/10.1016/j.autcon.2009.02.001

[10] Eshtehardian, E., Afshar, A., Abbasnia, R. "Time-cost optimization: using GA and fuzzy sets theory for uncertainties in cost", Construction Management and Economics, 26(7), pp. 679-691, 2008.

https://doi.org/10.1080/01446190802036128

[11] Kaveh, A. "Advances in Metaheuristic Algorithms for Optimal Design of Structures", Springer, Cham, Switzerland, 2021. https://doi.org/10.1007/978-3-319-46173-1

[12] Kaveh, A., Ilchi Ghazaan, M. "A new meta-heuristic algorithm: vibrating particles system", Scientia Iranica, 24(2), pp. 551-566, 2017.

https://doi.org/10.24200/sci.2017.2417

[13] Kaveh, A., Vazirinia, Y. "Chaotic vibrating particles system for resource-constrained project scheduling problem", Scientia Iranica, pp. 1826-1842, 2020.

https://doi.org/10.24200/sci.2019.51415.2163

[14] Kaveh, A., Vazirinia, Y. "Construction site layout planning problem using metaheuristic algorithms: a comparative study", Iranian Journal of Science and Technology, Transactions of Civil Engineering, 43(2), pp. 105-115, 2019. https://doi.org/10.1007/s40996-018-0148-6

[15] Goldberg, D. E. "Genetic Algorithms in Search, Optimization and Machine Learning", Addison-Wesley Longman, Boston, MA, USA, 1989.

https://doi.org/10.5860/choice.27-0936

[16] Eberhart, R., Kennedy, J. "A new optimizer using particle swarm theory", presented at MHS'95 Sixth International Symposium on Micro Machine and Human Science, Nagoya, Japan, Oct. 4-6, 1995. https://doi.org/10.1109/MHS.1995.494215

[17] Kaveh, A., Mahdavi, V. R. "Colliding bodies optimization: a novel meta-heuristic method", Computers and Structures, 139, pp. 18-27, 2014.

https://doi.org/10.1016/j.compstruc.2014.04.005 
[18] Kaveh, A., Talatahari, S. "Geometry and topology optimization of geodesic domes using charged system search", Structural and Multidisciplinary Optimization, 43(2), pp. 215-229, 2011. https://doi.org/10.1007/s00158-010-0566-y

[19] Kalhor, E., Khanzadi, M., Eshtehardian, E., Afshar, A. "Stochastic time-cost optimization using non-dominated archiving ant colony approach", Automation in Construction, 20(8), pp. 1193-1203, 2011. https://doi.org/10.1016/j.autcon.2011.05.003

[20] Deb, K., Pratap, A., Agarwal, S., Meyarivan, T. "A fast and elitist multiobjective genetic algorithm: NSGA-II", IEEE Transactions on Evolutionary Computation, 6(2), pp. 182-197, 2002.

https://doi.org/10.1109/4235.996017

[21] Zitzler, E., Laumanns, M., Thiele, L. "SPEA2: Improving the strength Pareto evolutionary algorithm", ETH Zürich, Zurich, Switzerland, TIK-Report 103, 2001. https://doi.org/10.3929/ETHZ-A-004284029

[22] Knowles, J. D., Corne, D. W. "Approximating the Nondominated Front Using the Pareto Archived Evolution Strategy", Evolutionary Computation, 8(2), pp. 149-172, 2000. https://doi.org/10.1162/106365600568167

[23] Coello Coello, C. A., Lechuga, M. S. "MOPSO: a proposal for multiple objective particle swarm optimization", presented at 2002 Congress on Evolutionary Computation CEC'02, Honolulu, HI, USA, May, 12-17, 2002. https://doi.org/10.1109/CEC.2002.1004388

[24] Kaveh, A., Ilchi Ghazaan, M. "A new VPS-based algorithm for multi-objective optimization problems", Engineering with Computers, 36, pp. 1029-1040, 2020. https://doi.org/10.1007/s00366-019-00747-8

[25] Zheng, D. X. M., Ng, S. T., Kumaraswamy, M. M. "Applying a Genetic Algorithm-Based Multiobjective Approach for TimeCost Optimization", Journal of Construction Engineering and Management, 130(2), pp. 168-176, 2004. https://doi.org/10.1061/(ASCE)0733-9364(2004)130:2(168)

[26] Afshar, A., Ziaraty, A. K., Kaveh, A., Sharifi, F. "Nondominated archiving multicolony ant algorithm in time-cost trade-off optimization", Journal of Construction Engineering and Management, 135(7), pp. 668-674, 2009. https://doi.org/10.1061/(ASCE)0733-9364(2009)135:7(668)

[27] El-Rayes, K., Jun, D. H. "Optimizing resource leveling in construction projects", Journal of Construction Engineering and Management, 135(11), pp. 1172-1180, 2009. https://doi.org/10.1061/(ASCE)CO.1943-7862.0000097

[28] Ghoddousi, P., Eshtehardian, E., Jooybanpour, S., Javanmardi, A. "Multi-mode resource-constrained discrete time-cost-resource optimization in project scheduling using non-dominated sorting genetic algorithm", Automation in Construction, 30, pp. 216-227, 2013. https://doi.org/10.1016/j.autcon.2012.11.014
[29] Zheng, D. X., Ng, S. T. "Stochastic Time-Cost Optimization Model Incorporating Fuzzy Sets Theory and Nonreplaceable Front", Journal of Construction Engineering and Management, 131(2), pp. 176-186, 2005.

https://doi.org/10.1061/(ASCE)0733-9364(2005)131:2(176)

[30] Zahraie, B., Tavakolan, M. "Stochastic Time-Cost-Resource Utilization Optimization Using Nondominated Sorting Genetic Algorithm and Discrete Fuzzy Sets", Journal of Construction Engineering and Management, 135(11), pp. 1162-1171, 2009. https://doi.org/10.1061/(ASCE)CO.1943-7862.0000092

[31] Kaveh, A., Khanzadi, M., Alipour, M. "Fuzzy Resource Constraint Project Scheduling Problem Using CBO and CSS Algorithms", International Journal of Civil Engineering, 14(5), pp. 325-337, 2016. https://doi.org/10.1007/s40999-016-0031-4

[32] Zadeh, L. A. "Fuzzy sets", Information and Control, 8(3), pp. 338$353,1965$.

https://doi.org/10.1016/S0019-9958(65)90241-X

[33] Yen, J., Langari, R. "Fuzzy Logic: Intelligence, Control, and Information", Prentice Hall, Hoboken, NJ, USA, 1998.

[34] Lorterapong, P., Moselhi, O. "Project-Network Analysis Using Fuzzy Sets Theory", Journal of Construction Engineering and Management, 122(4), pp. 308-318, 1996. https://doi.org/10.1061/(ASCE)0733-9364(1996)122:4(308)

[35] Dong, W., Wong, F. "Fuzzy weighted averages and implementation of the extension principle", Fuzzy Sets and Systems, 21(2), pp. 183199, 1987. https://doi.org/10.1016/0165-0114(87)90163-1

[36] Kaveh, A., Ilchi Ghazaan, M. "Enhanced colliding bodies optimization for design problems with continuous and discrete variables", Advances in Engineering Software, 77, pp. 66-75, 2014. https://doi.org/10.1016/j.advengsoft.2014.08.003

[37] Kaveh, A., Rastegar Moghaddam, M., Khanzadi, M. "Efficient multi-objective optimization algorithms for construction site layout problem", Scientia Iranica, 25(4), pp. 2051-2062, 2018. https://doi.org/10.24200/sci.2017.4216

[38] Zimmermann, H.-J. "Fuzzy Set Theory - and Its Applications", Springer, Dordrecht, Netherlands, 2011. https://doi.org/10.1007/978-94-010-0646-0

[39] Chen, P.-H., Weng, H. "A two-phase GA model for resource-constrained project scheduling", Automation in Construction, 18(4), pp. 485-498, 2009. https://doi.org/10.1016/j.autcon.2008.11.003

[40] Math Works "MATLAB", Math Works Inc. Natick, MA, USA, 2018. [online] Available at: http://www.mathworks.com 